\title{
Thermodynamics of the Complex su(3) Toda Theory
}

\author{
H. Saleur ${ }^{1,2}$ and B. Wehefritz-Kaufmann ${ }^{1}$ \\ 1 Department of Physics, University of Southern California, Los Angeles, CA 90089-0484, USA \\ ${ }^{2}$ Laboratoire de Physique Théorique et Hautes Energies, Université Paris 6, Paris, France
}

We present the first computation of the thermodynamic properties of the complex $s u(3)$ Toda theory. This is possible thanks to a new string hypothesis, which involves bound states that are non self-conjugate solutions of the Bethe equations. Our method provides equivalently the solution of the $s u(3)$ generalization of the XXZ chain. In the repulsive regime, we confirm that the scattering theory proposed over the past few years - made only of solitons with non diagonal $S$ matrices - is complete. But we show that unitarity does not follow, contrary to early claims, eigenvalues of the monodromy matrix not being pure phases. In the attractive regime, we find that the proposed minimal solution of the bootstrap equations is actually far from being complete. We discuss some simple values of the couplings, where, instead of the few conjectured breathers, a very complex structure (involving $E_{6}$, or two $E_{8}$ ) of bound states is necessary to close the bootstrap.

PACS: 72.10.-d, 73.40.Gk

Integrable quantum field theories (QFTs) based on $s u(2)$ have been the subject of intensive studies over the past many years. New theoretical tools like the quantum Q-operators [1], the Destri De Vega and Klümper and Pearce equations [2], the connections with spectral determinant theory [3], or the relations with elliptic curves and duality [4] have revealed mathematical structures of remarkable depth; they have also made possible the computation of quantities of experimental interest - and typical of strong interactions - in various low dimensional condensed matter systems 顿.

Formal developments, as well as practical applications, would largely benefit from an extension of these results to the case of other Lie algebras, in particular $s u(n)$. The situation here is somewhat embarrassing, however. Although the pillars of the $s u(2)$ case - the $X X Z$ chain and the associated sine-Gordon model - have been under control for a long time, even the simplest $s u(3)$ case is only very partially understood.

One of the difficulties here - and, from the field theory point of view, one of the most interesting issues at stake has to do with unitarity. Indeed, the simplest integrable generalizations of the sine-Gordon model are the complex 17 affine $s u(n)$ Toda theories defined by the Lagrangian:

\footnotetext{
${ }^{1}$ Real Toda theories involve entirely different issues. For a recent review see [5].
}

$$
\mathcal{L}=\frac{1}{2}\left(\partial_{\mu} \phi^{a}\right)\left(\partial^{\mu} \phi^{a}\right)-\lambda \sum_{j=0}^{n} e^{i \beta \alpha_{j} . \phi}
$$

where $\lambda>0, \alpha_{1}, \ldots, \alpha_{n-1}$ form the root system of the classical lie algebra $a_{n-1}, \alpha_{0}=-\sum_{j=1}^{n-1} \alpha_{j}$ is the negative of the longest root. The conformal weights of the perturbing operator in (11) are $\Delta=\bar{\Delta}=\frac{\beta^{2}}{4 \pi}$. In the following, we shall parameterize $\Delta=\frac{t-1}{t}$. The theory described by (1) is obviously non unitary at the classical level. The fascinating possibility was raised [6.7] that it could nevertheless describe a unitary field theory in a sufficiently strong quantum regime. This possibility was ruled out in the interesting paper [8], and we confirm and extend their observations here.

From a practical point of view, unitarity is not such a key issue. In fact, the most interesting applications of complex Toda theories are potentially found in disordered systems of statistical mechanics, where Toda theories based on superalgebras naturally seem to appear [9,10], leading most likely to even stronger violations of unitarity. More crucial then are the questions of completeness of the bootstrap, the physical meaning of the bound states, and the calculation of physical quantities.

The main progress in the study of complex Toda theories have been based on non perturbative $\mathrm{S}$ matrix analysis, following the pioneering work of [6]. One of the difficulties in this approach for $s u(n)$ is the appearance of a large number of poles in the $\mathrm{S}$ matrix elements, whose signification is not entirely clear: it was argued, after careful analysis of several cases, that most of these poles were not physical, and occurred rather by mechanisms generalizing Coleman Thun's [1]].

The issue of the completeness of the bootstrap in [6. [7] could be settled by a study of the thermodynamics, and a computation of the central charge in the UV, using the thermodynamic Bethe ansatz (TBA) [12] 2]. However, for the imaginary affine Toda theories (or, equivalently, the corresponding anisotropic quantum spin chains), the TBA has never been written so far, because of the complexity of the set of solutions: no natural "string hypothesis" had been proposed, up to now.

In this letter, we present the first solution of this vexing problem in the case of $s u(3)$. This allows us, in par-

\footnotetext{
${ }^{2}$ The approach developed in 20 bypasses some difficulties in the study of the thermodynamics, but does not give much information on the spectrum itself [13.
} 
ticular, to show that the quantum theory defined in (11) for $n=3$ is never unitary, even in the strong quantum regime, and that it presents considerably more bound states than expected.

Our main technical progress is an understanding of the solutions of the Bethe equations for systems based on $s u(3)$. Except in the exactly symmetric case, these solutions (the equivalent of the string hypothesis for the XXZ chain) had never been found. We hope that their understanding will spur new development in the area: generalizations of the works mentioned in the introduction, as well a calculations of physical properties in the super Toda case, seem particularly timely.

The problem we want to tackle is largely equivalent to the $s u(3)$ generalization of the XXZ spin chain. This integrable chain has been known for a long time 14, and reads

$$
\begin{array}{r}
H=-\sum_{j}\left[\sum_{r, s=1, r \neq s}^{3} e_{j}^{r s} e_{j+1}^{s r}+\cos \gamma \sum_{r=1}^{3} e_{j}^{r r} e_{j+1}^{r r}+\right. \\
\left.i \sin \gamma \sum_{r, s=1}^{3} \operatorname{sign}(r-s) e_{j}^{r r} e_{j+1}^{s s}\right]
\end{array}
$$

Relations of this system with the quantum field theory are two-fold. At the physical level, there is a simple integrable perturbation - obtained, in the quantum inverse scattering framework, by introducing heterogeneities of the spectral parameter, which amount to a staggered interaction - giving rise to (1) in the continuum limit, as can easily been shown using bosonization, or other arguments [15]. In that correspondence, $\gamma=\frac{\pi}{t}$. At a more formal level, observe first that the scattering matrices proposed in [16] are non diagonal. To study the thermodynamics of the gas of excitations in the S matrix approach, one needs to write wave functions, and impose their periodicity. This condition involves "passing" a particle through a set of other particles with which it scatters non diagonally: the phases obtained in this way are, in the inverse quantum scattering framework, eigenvalues of a monodromy matrix [17]. This monodromy matrix is nothing but the transfer matrix associated with the hamiltonian (2) (actually, it involves a slight generalization of this hamiltonian with a mixture of the fundamental representation and its conjugate), with however a renormalization of the anisotropy parameter, which becomes $\gamma=\frac{\pi}{t-1}$. The case $\gamma=\frac{\pi}{2}$, for instance (the equivalent of the XX chain, which is not a free problem here however), is a lattice regularization of the Toda theory for $\Delta=\frac{1}{2}$; it also turns out in the monodromy problem for the Toda theory at $\Delta=\frac{2}{3}$.

This double correspondence is very useful. If one is able to solve say the field theory problem for a particular value of $\Delta=\frac{t-1}{t}$, one should also be able to diagonalize the lattice model for $\gamma=\frac{\pi}{t}$, since it is just a discretization of this quantum field theory. But knowing the spectrum of (2) for $\gamma=\frac{\pi}{t}$ in turn means knowing the spectrum of the monodromy matrix for this value of $\gamma$. This is nothing but being able to solve the Toda theory for $\Delta=\frac{t}{t+1}$ !

Rather than dwell into the lengthy technical details, we would like to discuss first the solution of the lattice model for $\gamma=\frac{\pi}{2}$. We consider the slightly more general hamiltonian, where there is an arbitrary mixture of the fundamental representation and its conjugate. The lattice Bethe equations are 18]

$$
\begin{aligned}
& \left(\frac{\sinh \frac{1}{2}\left(y_{j}+i \pi / 2\right)}{\sinh \frac{1}{2}\left(y_{j}-i \pi / 2\right)}\right)^{N_{\overline{3}}}=\prod_{z} \frac{\sinh \frac{1}{2}\left(y_{j}-z_{r}-i \pi / 2\right)}{\sinh \frac{1}{2}\left(y_{j}-z_{r}+i \pi / 2\right)} \\
& \left(\frac{\sinh \frac{1}{2}\left(z_{r}+i \pi / 2\right)}{\sinh \frac{1}{2}\left(z_{r}-i \pi / 2\right)}\right)^{N_{3}}=\prod_{y} \frac{\sinh \frac{1}{2}\left(z_{r}-y_{j}-i \pi / 2\right)}{\sinh \frac{1}{2}\left(z_{r}-y_{j}+i \pi / 2\right)}
\end{aligned}
$$

where we have not written a crucial but complicated sign on the right hand sides, and the energy reads $E=$ $-2 \sum \frac{1}{\cosh z}-2 \sum \frac{1}{\cosh y}$. A combination of numerical studies and analytical arguments led to the identification of the following sets of roots in the thermodynamic limit. The $y$ and $z$ can both be real, or both have an imaginary part equal to $\pi$ (antistring). In addition, it is possible to have complexes, with a two string $z$ centered on an antistring $y$, that is: $y=r+i \pi, z=r \pm i \frac{\pi}{2}$ (here $r$ is a real number, and we identified $r+3 i \frac{\pi}{2}$ and $\left.r-i \frac{\pi}{2}\right)$, and the same thing with $y$ and $z$ reversed. The existence of these complexes is easy to understand. Suppose for instance that $z$ has a positive imaginary part, so the lhs of the second equation in (3) blows up as $N_{3} \rightarrow \infty$. It is then necessary to have the rhs also blow up, which can be accomplished if there exists a $y$ such that $y=z+i \frac{\pi}{2}$. The same goes if $z$ has a negative imaginary part, so the complexes proposed are the minimal possible structures leading to real Bethe equations. Together with the real and antistring solutions, they reproduce the $3+3$ degeneracy expected for the fundamental solitons. In addition however, another type of complex (which we call $y z$ in the following) is possible, of the form $z=r+i \frac{3 \pi}{4}, y=r+i \frac{5 \pi}{4}$, together with the conjugate. Usually, one would expect that these two complexes actually come glued together to ensure reality of the Bethe equations and the eigenvalues, resulting in a sort of "quartet" 19. This does not seem to be the case here. Rather, to reproduce the correct entropy or central charge, one needs to treat their densities as independent.

These complexes are the ones which dominate the thermodynamics. As usual, there are many other solutions to the Bethe equations. The existence of solutions which are not invariant under complex conjugation being rather unusual, we illustrate it briefly. Figure 1 shows the $y z$ complex obtained by a numerical solution of (3) for $N_{\overline{3}}=0$ and for different values of $N_{3}$. In this example - which seems the simplest possible - the half sum of the imaginary parts of $y$ and $z$ goes to $\pi$ in the thermodynamic limit, but their difference does not go to $\frac{\pi}{2}$. We conjec- 
ture that this would however be the case for the overwhelming majority of such complexes: we checked that only in this case, are the correct scattering theory and thermodynamics recovered.

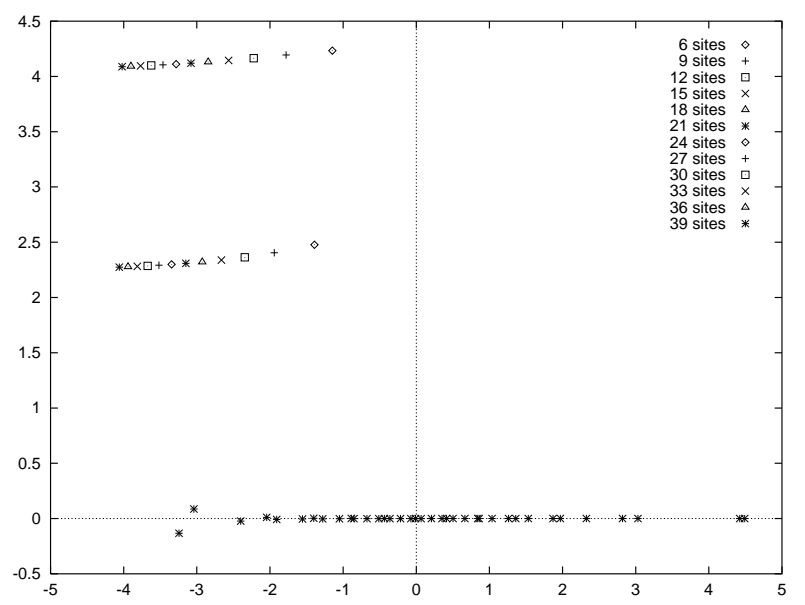

FIG. 1. A numerical solution of (3) containing a $y z$ complex for $N_{\overline{3}}=0$ for different values of $N_{3}=6,9, \cdots, 39$. For 39 sites we show all roots belonging to this particular state, whereas for all other lattice lengths only the $y z$ complex is shown.

The lattice model without heterogeneities has a continuum limit which is a conformal field theory, made of two bosons compactified on a triangular lattice. The latter is unitary, and we have checked numerically that the imaginary parts of the eigenenergies of (2) scale to zero faster than their real parts, ensuring reality of the conformal weights, and, actually, unitarity of the conformal field theory, despite the non-hermicity of the hamiltonian. Meanwhile, the lattice model with heterogeneities does have a continuum limit described by a complex Toda theory, with $\Delta=\frac{1}{2}$. In this case, the complexes correspond to physical particles, the bound states discussed in [6,, 4 . The fact that they are described by solutions of the Bethe ansatz which are not self conjugate corresponds to the shocking fact that, in the quantum field theory, their $\mathrm{S}$ matrix is not a pure phase (and "naive" unitarity, in the sense of the scattering theory, is broken).

Accordingly, a crucial feature related with the $y z$ complexes is that they give rise to non real kernels in the continuum Bethe ansatz. For instance, the kernel for the scattering of a real $z$ through a $y z$ complex $z=r+i \frac{3 \pi}{4}$, $y=r+i \frac{5 \pi}{4}$ is $\frac{1}{i} \frac{d}{d z} \ln \Phi=-1 / \cosh \left(z-r+\frac{i \pi}{4}\right)$. Although at equilibrium the densities of the two types of conjugate complexes are equal, it is necessary to let them vary independently to get the correct entropy. The physical results will be the same as the ones that would be obtained with a different theory, where the scattering kernels would be real, and given by the real part of the true kernels. This is what we will consider in the following, to make the notations simpler.

The lattice model whose equations are (3) occurs also in the problem of diagonalizing the monodromy matrix for $\Delta=\frac{2}{3}$. The complexes just discussed have no physical meaning then: they just correspond to pseudo particles of zero mass. However, the fact that they are not self conjugate means that eigenvalues of the monodromy matrix will not, in general, have modulus one. Contrary to early claims [6], unitarity is strongly violated in the theory, even in the absence of bound states. The physical implications of having "monodromies" which are not pure phases are not clear to us: presumably $S$ matrix elements have to be considered as formal objects used to build wave functions, and cannot be given a reasonably meaning in terms of scattering processes is.

This basic structure generalizes easily to the case $t$ integer. For $\gamma=\frac{\pi}{t}$, the Bethe equations contain in addition a term of interaction between $y$ roots, and between $z$ roots. The solutions are the usual $y$ or $z$ real, antistrings, or $2, \ldots, t-1$ strings, plus $y$ - $t$ strings centered on $z$ antistrings, and the same thing with $y$ and $z$ reversed. In addition, the $y z$ complexes now are of the form $z=r+i \pi-i \frac{\pi}{2 t}, y=r+i \pi+i \frac{\pi}{2 t}$, and the same with $y$ and $z$ exchanged.

To proceed, one has to use these results to solve the monodromy problem. At $\Delta=\frac{t-1}{t}$, passing a soliton through a set of $N_{3}, N_{\overline{3}}$ solitons at various rapidities gives rise to a "phase" which is an eigenvalue of the monodromy matrix. The latter is essentially the transfer matrix associated with (2), but for $\gamma=\frac{\pi}{t-1}$. Its eigenvalues are obtained by generalizing slightly the Bethe ansatz equations, so the arguments in the lhs of (3) are shifted by the corresponding rapidities, and using the generalized string hypothesis described previously.

A few technical details are involved, which are completely equivalent to what happens in the $s u(2)$ case [20]. We will only describe the end result, which is quite simple. For the case $t \rightarrow \infty$, the TBA has been known for a long time 21,22, and has a structure that mimics the known one for $s u(2)$, with an infinity of massless nodes corresponding to the usual strings of the Bethe equations. Like for the $s u(2)$ case, the introduction of anisotropy truncates this to a finite number of strings: the truncation has to be completed by the proper "end structure" of the diagram. Like in the $s u(2)$ case, this structure,

\footnotetext{
${ }^{3}$ A somewhat related problem occurs for instance in the massless description of conformal field theories.
} 
for $s u(3)$, is given by the Bethe roots for $\gamma=\frac{\pi}{2}$. After some manipulations, the results are the following. We call $\sigma, \sigma^{h}$ the densities of the solitons at rapidity $\theta$, and $m$ the mass of the fundamental soliton. Then

$$
\begin{gathered}
\sigma^{3}+\sigma^{3 h}=m \cosh \theta+\phi \star\left(\rho_{1}^{3 h}+\sigma^{\overline{3} h}\right) \\
\ldots \\
\rho_{n}^{3}+\rho_{n}^{3 h}=\phi \star\left(\rho_{n-1}^{3 h}+\rho_{n+1}^{3 h}+\rho_{n}^{\overline{3}}\right) \ldots
\end{gathered}
$$

and similarly for $\overline{3}$, with $\rho_{0} \equiv \sigma$. We recognize here the standard equations for the minimal model [16]. In addition, in this untruncated case, we need the "closure" relations, which are the key to the whole problem. They read here

$$
\begin{aligned}
\rho_{t-3}^{3}+\rho_{t-3}^{3 h} & =\phi \star\left(\rho_{t-4}^{3 h}+\rho_{t-2}^{3 h}+\rho_{t-3}^{\overline{3}}+\rho_{a_{1}}^{3}+\rho_{a_{2}}^{3}\right) \\
& +\psi \star\left(\rho_{b}^{3}+\rho_{b}^{\overline{3}}\right) \\
\rho_{t-2}^{3}+\rho_{t-2}^{3 h} & =\phi \star\left(\rho_{t-3}^{3 h}+\rho_{t-2}^{\overline{3}}-\rho_{a_{1}}^{\overline{3}}-\rho_{a_{2}}^{\overline{3}}\right) \\
& -\psi \star\left(\rho_{b}^{3}+\rho_{b}^{\overline{3}}\right) \\
\rho_{a_{i}}^{3}+\rho_{a_{i}}^{3 h} & =\rho_{t-2}^{3}+\rho_{t-2}^{3 h}
\end{aligned}
$$

and similarly for $\overline{3}$; finally

$$
\begin{aligned}
\rho_{b}^{3}+\rho_{b}^{3 h} & =\psi \star\left(\rho_{t-3}^{3 h}+\rho_{t-2}^{3}-\rho_{a_{1}}^{3}-\rho_{a_{2}}^{3}+3 \rightarrow \overline{3}\right) \\
& -\phi \star\left(\rho_{b}^{3}+\rho_{b}^{\overline{3}}\right)
\end{aligned}
$$

In these equations, the kernels are defined by their fourier transforms $\hat{\phi}=\frac{1}{2 \cosh x}$ and $\hat{\psi}=\frac{\cosh x / 2}{2 \cosh x}$. The subscripts $a_{i}, i=1,2$ stand for antistrings or $t$ strings centered on antistrings; $b$ stands for $y z$ complexes.

We set $e^{-\epsilon_{0} / T}=\sigma / \sigma^{h}, e^{-\epsilon_{n} / T}=\rho_{i}^{h} / \rho_{i}, n=1, \ldots, t-$ $2, e^{-\epsilon_{a} / T}=\rho_{a_{i}} / \rho_{a_{i}}^{h}, e^{-\epsilon_{b} / T}=\rho_{b} / \rho_{b}^{h}$ (color labels are kept implicit here). Introducing the usual variables $x_{\alpha}=$ $e^{-\epsilon_{\alpha} / T}$, the TBA in the UV limit reduces to the system $\left(x_{t-2}=1 / x_{a}\right)$ :

$$
\begin{array}{rrr}
x_{0}= & \left(1+x_{1}\right)^{1 / 2}\left(1+\frac{1}{x_{0}}\right)^{-1 / 2} \\
\ldots & \\
x_{n}= & \left(1+x_{n-1}\right)^{1 / 2}\left(1+x_{n+1}\right)^{1 / 2}\left(1+\frac{1}{x_{n}}\right)^{-1 / 2} \\
\ldots & \\
x_{t-3}= & \left(1+x_{t-4}\right)^{1 / 2}\left(1+x_{a}\right)^{3 / 2}\left(1+\frac{1}{x_{t-3}}\right)^{-1 / 2}\left(1+x_{b}\right) \\
x_{a}= & \left(1+x_{t-3}\right)^{1 / 2}\left(1+\frac{1}{x_{a}}\right)^{-1 / 2}\left(1+x_{a}\right)^{-1}\left(1+x_{b}\right)^{-1} \\
x_{b}= & \left(1+x_{t-3}\right)\left(1+\frac{1}{x_{a}}\right)^{-1}\left(1+x_{a}\right)^{-2}\left(1+x_{b}\right)^{-1}
\end{array}
$$

The solution of this system is

\footnotetext{
${ }^{4}$ We define $\hat{f}(x)=\frac{1}{2 \pi} \int f(\theta) e^{-i n x \theta / \pi} d \theta$.
}

$$
\begin{aligned}
& x_{j}=\frac{(j+1)(j+4)}{2}, j=0, \ldots, t-3 \\
& x_{a}=\frac{t-1}{t+1} \\
& x_{b}=\frac{(t-1)^{2}}{4 t}
\end{aligned}
$$

In the IR limit, we get an identical TBA, but with the replacement $t \rightarrow t-1$ exactly as in the $S U(2)$ case [20], because the first two nodes become infinitely massive, $\epsilon_{0}=\infty$. The whole TBA system is actually quite similar to the one for the sine-Gordon model: the "left part" can be conveniently encoded in a ladder diagram with base the Dynkin diagram of $a_{2}$, and in the isotropic limit $t \rightarrow \infty$, this is all that matters. For finite $t$ however, the diagram has to be closed to the right, and the closing terms are more complicated than for sine-Gordon: in addition to $3+3$ nodes standing algebraically for the representations 3 and $\overline{3}$ (in the sine-Gordon case, there are two such nodes corresponding to the self-conjugate fundamental representation), the closure requires the nodes associated with the non real solutions of the Bethe ansatz, whose algebraic meaning has eluded us up to now. The TBA diagrams for $\mathrm{SU}(2)$ and $\mathrm{SU}(3)$ are shown in Fig. 2.
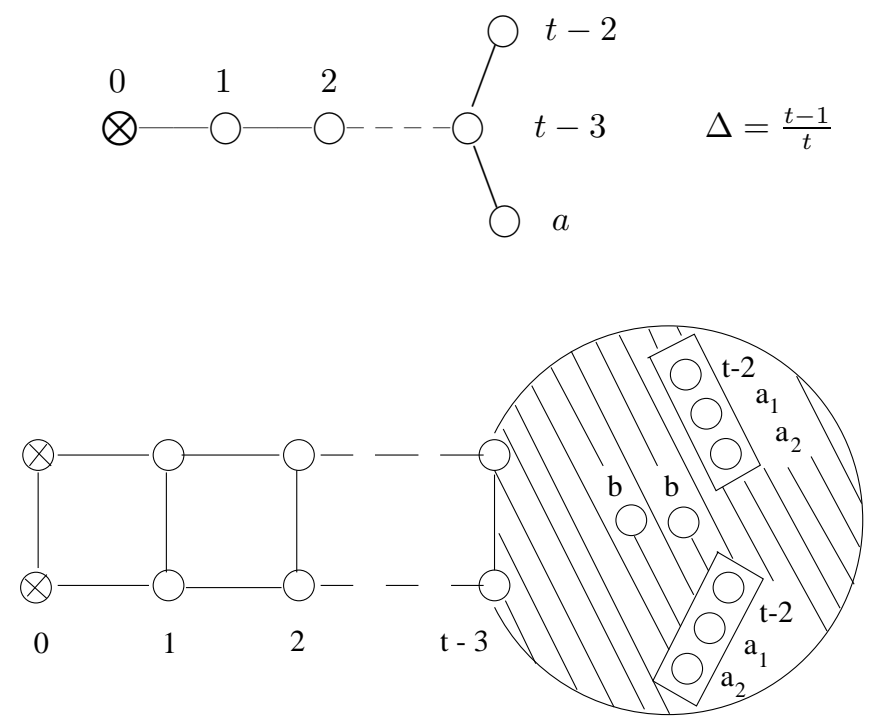

FIG. 2. The TBA diagrams for $S U(2)$ and $S U(3)$

Using the basic identity [23]: $L(x)+L(y)=L(x y)+$ $L\left[\frac{x(1-y)}{1-x y}\right]+L\left[\frac{y(1-x)}{1-x y}\right]$, one finds

$$
\begin{gathered}
\sum_{j=0}^{t-3} 2 L\left[\frac{j^{2}+5 j+4}{j^{2}+5 j+6}\right]+6 L\left[\frac{t-1}{2 t}\right] \\
+2 L\left[\left(\frac{t-1}{t+1}\right)^{2}\right]=(t-1) \frac{\pi^{2}}{3},
\end{gathered}
$$

and therefore, $c=2(t-1)-2(t-2)=2$, as required. Note that for the sine-Gordon case, the sum of dilogarithms for the UV (IR) diagram was $(t-1) \frac{\pi^{2}}{6}($ resp. $t-2)$ instead, 
generalizing nicely the combinatorics to the case of rank two.

Still like in the sine-Gordon case, the same TBA diagram supports the scattering theory for generalized supersymmetric extensions: by putting the mass term on the $k^{t h}$ node, the central charge now becomes $c=\frac{8 k}{k+3}$, in agreement with the central charge for $S U(3)_{k}$ theories.

The Toda theory can be twisted by coupling a charge to the total diagonal $U(1)$ current. The kinks come in the form of 3 and $\overline{3}$, and the total charge can be recast in terms of the solutions of the associated, non diagonal scattering problem, as $Q \propto(t-1) \int \rho_{t-2}^{3}-\rho_{a_{1}}^{3}+(3 \rightarrow \overline{3})$. The thermodynamics is more complicated to carry out than in the sine-Gordon case however, densities of holes and particles mixing up in a complex fashion in the right hand side of the universal Bethe equations. It can still be shown that, with a fugacity -1 for the solitons, the diagram truncates, leaving only the nodes $j=0, \ldots, t-5$, for $t \geq 5$, the well known result for the minimal model $S U(3)_{1} \times S U(3)_{t-4} / S U(3)_{t-3}$. Note that more nodes disappear than in the sine-Gordon case: the case where the minimal theory has $c=0$ now corresponds to a dimension $\Delta=\frac{3}{4}$ in the Toda theory.

The study of the points $\Delta=\frac{t-1}{t}, t$ integer, therefore presents few surprises, except maybe the non unitarity, due to the complex solutions of the Bethe equations. We do not expect a qualitatively different behaviour when $t$ is not an integer, provided $\Delta$ lies in the repulsive regime, $\Delta \geq 3 / 5$, where no bound states are expected. In contrast, the attractive regime turns out to be considerably more complicated.

From a technical point of view, the points $\Delta=\frac{1}{t}$, could be studied as the continuum limit of the chain with $\gamma=\frac{\pi}{t}$ still, but with the opposite sign of the hamiltonian. This observation is crucial in the case of the XXZ chain, and allows one to essentially use the same type of solutions of the Bethe equations to study both the attractive and the repulsive regime: it is well known that the TBAs for these two cases are very closely related. In the $s u(3)$ case, the situation is more involved: depending on the sign of the hamiltonian, it is not the same type of solutions of the Bethe equations that determines the thermodynamics. This can be illustrated in the case $\gamma=\frac{\pi}{2}$. Taking say a chain made only of 3's, the ground state is $y$ and $z$ real for one sign, $y$ antistring and $z$ real for the other. This still is like for the XX chain. However, the other solutions of the Bethe equations are different: for instance, instead of having two string $z$ centered on antistrings $y$ 's, one has now two string $z$ centered on real $y$ 's. Things get worse as $t$ increases, and solutions that were possible but did not contribute to the thermodynamics for one sign of the coupling, start being important for the other.

The net result is that we have not been able to reproduce the simple $c=2$ result for any point in the attractive regime except the $\Delta=\frac{1}{2}$ case. To illustrate the nature of the difficulties, let us concentrate for a while on the theories for which $\lambda=\frac{4 \pi}{\beta^{2}}-1$ is of the form $\lambda=\frac{2 n+1}{3}$, or $\lambda=\frac{2 n+2}{3}$. In that case, the deformation parameter for the quantum group symmetry of the scattering theory, $q=-\exp (i \pi \lambda)$ is a cubic root of unity, and the q-dimension of the 3 or the $\overline{3}$ vanish exactly, $(3)_{q}=0$. This means that the RSOS truncation 24,25] of the theory is very simple: the kink part entirely disappears from the scattering, and one is left with a diagonal scattering for the scalar bound states. This scattering leads to straightforward TBA calculations, which one can compare against the predictions of conformal field theory. The disagreement with the scattering theory proposed in [7] is considerable. Let us give two examples. For $\lambda=\frac{4}{3}$, the analysis of [7] predicts only a pair of bound states: to reproduce the effective central charge $c_{\text {eff }}=6 / 7$ together with its apparent conformal weight $\Delta_{\text {eff }}=1 / 7$, one needs in fact 6 bound states, essentially reproducing the well known $E_{6}$ scattering theory 26]. Note that in this theory, there are pairs of conjugate particles, but also self-conjugate ones: the issue of whether bound states in $s u(3)$ should always appear in pairs is not clear, and this counter example demonstrates it is not always the case.

If the appearance of $E_{6}$ is somewhat surprising, an even bigger surprise is encountered for $\lambda=\frac{5}{3}$. In this case, the effective central charge is $c_{\text {eff }}=1$, and the apparent weight $\Delta_{\text {eff }}=1 / 16$. The scattering theory in [7] predicts two pairs of bound states, i. e. 4 scalar particles. To close the bootstrap, it turns out that one needs in fact 16 particles, with a scattering theory that is a sort of double $E_{8}$ [27]: if one labels the $E_{8}$ particles by $j$, the $s u(3)$ particles by $B_{j}$, then one has the identity $S_{B_{j} \bar{B}_{j}} S_{B_{k} \bar{B}_{k}}=$ $S_{j k}$ : this guarantees that the central charge in the UV is $2 \times \frac{1}{2}=1$ f , as required.

The ratio of the actual number of bound states and the minimal one is actually growing very quickly, and it is not clear what to do to close the bootstrap even for the simplest non trivial reflectionless point, $\Delta=\frac{1}{3}$, where the conjectured scattering theory [7.28, involving the fundamental solitons $A_{0}, \bar{A}_{0}$, excited solitons $A_{1}, \bar{A}_{1}$, and breathers $B_{1}, \bar{B}_{1}, B_{2}, \bar{B}_{2}$, gives only $c=1.90821$. Note that this point is quite special, since the particles can be gathered into 2 eightuplets of particles of mass $M$ and $\sqrt{3} M$, indicating the presence of a hidden $D_{4}^{(3)}$ symmetry 29,30. Introducing more particles increases $c$, but we haven't found a closure of the bootstrap, even after introducing large numbers of bound states 5 .

The repulsive regime for $s u(n), n \geq 4$, presents similar

\footnotetext{
${ }^{5}$ This doubling is similar to what happens in the scattering theory for the 3 state Potts model, with $c=4 / 5$, compared with the scattering theory for the Yang Lee model, with $c_{\text {eff }}=$ $2 / 5$.

${ }^{6}$ Let us stress here that the possibility of having missed some CDD factors in the original scatterings should be considered
} 
difficulties: the scattering theory of [7,28] is incomplete even at the point $\Delta=\frac{1}{2}$, and there again, more bound states have to be introduced.

In conclusion, except for the simplest case of $s u(3)$ in the repulsive regime, the scattering theory of the complex Toda theories is not under control, in our opinion. The large body of literature on the subject (see e. g. [32] for some recent developments) has identified a minimal structure for it, but reality is more complicated (a fact well known to some experts). How complicated is not clear to us: without an algebraic understanding of the bound states, tackling the general case is a very laborious task, and it is not even clear that the bootstrap will close with a finite number of bound states, except for some special values: clearly, more work is needed in this direction.

Meanwhile, the solution of the $s u(3)$ theory in the repulsive regime opens the way to several problems of physical interest, in particular study of $s u(3)$ anisotropic Kondo problems, and su(3) tunneling problems, on which we hope to report soon.

Acknowledgments: we thank P. Fendley for very useful discussions and G. Takacs for comments on the manuscript. The work was supported by the DOE and the NSF (under the NYI program). B. W.-K. acknowledges support from the Deutsche Forschungsgemeinschaft (DFG) under the contract KA 1574/1-1.

[1] V.V. Bazhanov, S. L. Lukyanov, and A. B. Zamolodchikov, Comm. Math. Phys. 200 (1999) 297.

[2] P.A. Pearce and A. Klümper, Phys. Rev. Lett. 68 (1991) 97; C. Destri and H. de Vega, Phys. Rev. Lett. 69 (1992) 2313.

[3] P. Dorey and R. Tateo, J. Phys. A32 (1999) L 419.

[4] P. Fendley and H. Saleur, Phys. Rev. Lett. 81 (1998) 2518.

[5] P. Christe and G. Mussardo, Int. J. Mod. Phys. A5 (1990) 4581; E. Corrigan, "Recent developments in affine Toda quantum field theory", hep-th/9412213.

[6] T.J. Hollowood, Int. J. Mod. Phys. A8 (1993) 947; hepth/9203076.

[7] G. M. Gandenberger, Nucl. Phys. B449 (1995) 375; hepth/9501136.

[8] G. Takacs and G.M.T. Watts, Nucl. Phys. B547 (1999) 538.

[9] S. Guruswamy, A. Leclair and A.W.W. Ludwig,

as excluded, since our lattice calculations (see also [31]) do fully reproduce the accepted kernels.
" $G L(N / N)$ super current-algebras for disordered systems", cond-mat/9909143.

[10] Z.S. Bassi and A. Leclair, "The exact S matrix for an $\operatorname{osp}(2 / 2)$ disordered system", hep-th/9911105.

[11] S. Coleman and H.J. Thun, Comm. Math. Phys. 61 (1978) 31.

[12] A. B. Zamolodchikov, Nuc. Phys. B342 (1990) 695.

[13] P. Zinn-Justin, J. Phys. A31 (1998) 6747.

[14] O. Babelon, H.J. de Vega, and C.M. Viallet, Nucl. Phys. B200 (1982) 266; Nucl. Phys. B220 (1983) 283.

[15] C. Destri and H. de Vega, J. Phys. A22 (1989) 1329; N. Yu Reshetikhin and H. Saleur, Nucl. Phys. B419 (1994) 507.

[16] T.J. Hollowood, Phys. Lett. B320 (1994) 43.

[17] P. Fendley and K. Intrilligator, Nucl. Phys. B380 (1992) 265.

[18] J. Abad and M. Rios, Phys. Rev. B53 (1995) 14000; J. Phys. A29 (1996) L1.

[19] O. Babelon, H.J. de Vega, and C.M. Viallet, Nucl. Phys. B220 (1983) 13.

[20] P. Fendley and H. Saleur, Nucl. Phys. B388 (1992) 609.

[21] N. Andrei, K. Furuya and J. H. Lowenstein, Rev. Mod. Phys. 55 (1983) 331; A.M. Tsvelik and P.B. Wiegmann, Adv. Phys. 32 (1983) 453.

[22] F. Ravanini, R. Tateo, and A. Valleriani, Int. J. Mod. Phys. A8 (1993) 1707.

[23] A. Kirillov, "Dilogarithmic identities", hep-th/9408113.

[24] N. Yu Reshetikhin and F. Smirnov, Comm. Math. Phys. 131 (1990) 157.

[25] C. Ahn, D. Bernard and A. Leclair, Nucl. Phys. B346 (1990) 409.

[26] G. Sotkov and C.J. Zhu, Phys. Lett. B229 (1989) 391.

[27] A. B. Zamolodchikov, Int. J. Mod. Phys. A3 (1988) 743.

[28] T.J. Hollowood, Nucl. Phys. B384 (1992) 523.

[29] I. Vaysburd, "Integrable perturbations of $W_{n}$ and WZW models", hep-th/9402061.

[30] G. Takacs, Nucl. Phys. B502 (1997) 629; hep-th/9701118.

[31] A. Doikou and R. I. Nepomechie, "Soliton S-matrices for the critical $A_{N-1}^{(1)}$ chain"; hep-th/9906069.

[32] G. M. Gandenberger and N. J. Mac Kay, Nucl. Phys. B457 (1995) 240; G. M. Gandenberger, N. J. MacKay and G. M. T. Watts, Nucl. Phys. B65 (1996) 329. 